\title{
INSIGHTS INTO THE CONDENSATION OF PAHS IN THE ENVELOPE OF IRC +10216
}

\author{
L. Biennier ${ }^{1}$, H. Sabbah ${ }^{1,2}$, S.J. Klippenstein ${ }^{3}$, V. Chandrasekaran ${ }^{1,4}$, \\ I.R. Sims ${ }^{1}$ and B.R. Rowe ${ }^{1}$
}

\begin{abstract}
The mechanisms of nucleation and growth of carbon dust particles in circumstellar envelopes of carbon-rich stars in the red giant and AGB phases of their evolution are poorly understood. It has been proposed that the transition of gas phase species to solid particles, is achieved by the formation of a critical nucleus composed of two PAHs held together by van der Waals forces. Some insights into the validity of the nucleation of PAH molecules in the envelope can be gained through the investigation of the thermodynamics of dimers, representing the first stage towards condensation. We have performed experiments to identify the temperature range over which small PAH clusters form in saturated uniform supersonic flows. The kinetics of the formation has also been investigated. The experimental data have been combined with theoretical calculations. We unambiguously demonstrate that the association of small PAHs such as pyrene $\left(\mathrm{C}_{16} \mathrm{H}_{10}\right)$ is slower than the destruction of the dimer in warm and hot environments such as IRC +10216 . Our findings challenge a formation model based on the physical stacking of small PAH units in circumstellar shells of carbon rich stars.
\end{abstract}

\section{Introduction}

The outflows of carbon rich stars in their asymptotic giant branch phase (AGB) are the primary sources of carbon dust (Henning \& Salama 1998). AGB stars lose up to $80 \%$ of their original mass in the form of an envelope, with typical loss

\footnotetext{
${ }^{1}$ Institut de Physique de Rennes, Équipe: "Astrochimie Expérimentale", UMR CNRS 6251, Université de Rennes 1, Campus de Beaulieu, 35042 Rennes Cedex, France

2 Current address: Zarelab, Chemistry Department, Stanford University, CA 94305, USA

3 Chemical Sciences and Engineering Division, Argonne National Laboratory, Argonne, IL 60439, USA

4 Current address: Institut des Sciences Moléculaires, UMR CNRS 5255, Université Bordeaux 1, 33405 Talence, France
} 
rates of $10^{-6}$ to $10^{-4} M_{\odot} \mathrm{yr}^{-1}$. There is limited observational evidence for the formation of dust in other sources, such as Supernovae (SNe) and novae, but their relative importance as sources of interstellar dust, are still uncertain (Jones 2005).

The determination of the physical conditions under which dust is generated is critical. Estimates of the temperature and pressure of circumstellar envelopes strongly depend on various model assumptions, such as mass loss rates, gas density outflow, and velocity. Observations have revealed that the envelope of evolved stars is huge, extending over $10^{4}$ to $10^{5}$ stellar radii. Close to the photosphere $\left(<5 R_{\star}\right)$, the gas is dense $\left(n \geq 10^{10}\right.$ molecule $\left.\cdot \mathrm{cm}^{-3}\right)$ and warm $(T \sim 1500 \mathrm{~K})$. As the matter flows away from the star, it expands $\left(n \leq 10^{5}\right.$ molecule $\left.\cdot \mathrm{cm}^{-3}\right)$ and cools rapidly down to $25 \mathrm{~K}$ at several hundreds of $R_{\star}$ (Ziurys 2006).

The most refractory material composing the atmosphere, such as carbides, nucleates first in the innermost region, even inside the photosphere. Once formed, they act as nuclei sites for heterogeneous condensation of more volatile carbonaceous molecules. C condensation is delayed until homogeneous nucleation can occur at higher supersaturation levels farther out from the star (Bernatowicz et al. 2006). Some complexity is added to this already debated picture, by the propagation of shock waves, produced by stellar pulsations, which greatly enhance the density and temperature just after the shock front. Because of the variety of physical conditions, the chemistry varies substantially throughout the envelope. In the inner shell $\left(<\right.$ a few $R_{\star}$ ), the temperature and density are sufficiently high that thermodynamic equilibrium governs molecular abundances of a number of molecules (Ziurys 2006). This assumption is confirmed by astronomical observations in the infrared, which have shown that stable, closed-shell species such as $\mathrm{CS}, \mathrm{NH}_{3}, \mathrm{HCN}$, and $\mathrm{HCCH}$ have high abundances in the inner envelope of the well studied $\mathrm{IRC}+10216$ carbon star $\left(\approx 10^{-3}\right.$ to $10^{-6}$, relative to $\left.\mathrm{H}_{2}\right)$.

As the density falls with radius, interstellar UV photons penetrate the atmosphere and generate reactive species through photodissociation that subsequently participate in a complex gas-phase chemistry to produce daughter molecules in the outer envelope. Interferometer maps of IRC +10216 reveal radicals such as $\mathrm{CN}, \mathrm{CCH}, \mathrm{C}_{3} \mathrm{H}$, and $\mathrm{C}_{4} \mathrm{H}$ organized in shell-like structures near the envelope edge (Guelin et al. 1993).

It is generally accepted that the production pathway determines the resulting structure, chemical composition, and morphology of the grains (Jager et al. 2009). However, the formation pathways of carbonaceous grains from molecular components and clusters, evoked by Jager in this volume (2010), are not well understood. From a chemical viewpoint, the high abundance of hot acetylene (Fonfria et al. 2008) and the scarcity of oxydizing molecules in circumstellar envelopes of carbon rich stars are expected to lead to the production of carbonaceous particles through processes similar to the ones encountered in terrestrial low pressure flames. The analogy strongly suggests the presence of Polycyclic Aromatic Hydrocarbons (PAHs) as building blocks, intermediates or side products in the generation of carbon dust particles. Remarkably, the aromatic IR emission features, characteristic of PAHs, are generally not seen in the spectra of carbon-rich AGB stars, but are systematically detected in carbon-rich post-AGB objects and 
Planetary Nebulae. Two interpretations are contending to explain the infrared emission features in the spectra of the latter sources. It can be the consequence of efficient pumping arising from the increased effective temperature of the central object as it loses its remnant envelope and shifts to the blue in the HertzsprungRussell diagram on its way to becoming a white dwarf. Alternatively, it may be the result of photochemical processing of hydrogenated amorphous carbon (HAC) material formed in the outflows. The ground-based and ISO/SWS detection of the aromatic infrared emission features in the spectra of the carbon-rich AGB star TU Tau (Boersma et al. 2006), likely irradiated by its blue binary companion, does not provide an unambiguous argument in favor of either of the scenarios. Independent of the formation pathways actually followed, the presence of PAH features in the IR spectra of carbon-rich post-AGB objects and planetary nebulae (PNe) lends indirect support for the importance of late-type stars as sources for PAHs in the interstellar medium (Tielens 2008).

Theoretical studies on the chemical pathways towards PAHs in circumstellar envelopes are reviewed in this conference by Cherchneff (2010). Briefly, the formation of PAHs from small gaseous hydrocarbons in the circumstellar envelopes was initially proposed by Frenklach \& Feigelson (1989b). Their study was the first to introduce chemical kinetics in those atmospheres. The chemical scheme considered was largely inspired from combustion studies and relies heavily on the presence of acetylene $\mathrm{C}_{2} \mathrm{H}_{2}$ and radicals such as $\mathrm{C}_{2} \mathrm{H}$. Reactions involving oxygen were removed, whereas benzyne $\mathrm{C}_{6} \mathrm{H}_{4}$ was introduced in the network. The dominant $\mathrm{PAH}$ formation pathway was identified as the hydrogen abstraction acetylene addition (HACA) route. Cherchneff revised and extended the scheme, explicitly including radicals, such as $\mathrm{C}_{3} \mathrm{H}_{3}$ and aromatics up to $\mathrm{C}_{18} \mathrm{H}_{12}$ (Cherchneff et al. 1992). In the study, Cherchneff et al. considered that the gas density was so low that collisions did not couple efficiently the vibrational and translational degrees of freedom. As a consequence, $\mathrm{PAH}$ molecules did end up with vibrational temperatures significantly lower than gas kinetic temperature. Nevertheless, the conclusion was that PAH formation yields were very sensitive to gas density and temperature, and that they were much smaller than values inferred from the observed dust content of late type carbon rich stellar envelopes. They suggested that the PAHs may form instead very close to the photosphere. Allain et al. further explored the processes affecting PAH formation and growth (Allain et al. 1997). They carried out calculations of the PAH temperature by balancing heating via collisions with gas particles and absorption of stellar emission and cooling via emission from the rotational and vibrational levels. In their work they treated heating processes simultaneously and found that low PAH temperatures enable an efficient formation and growth of PAHs in the circumstellar envelopes of C-stars. The calculated PAH formation rate was large enough to support the idea that PAHs could constitute the first step in the formation of dust grains. The processes, either chemical or physical, that could explain the transition from these large molecules to small dust grains, were however not investigated. Nucleation triggered by association of PAH molecules formed in the gas phase, considered as a key step in controversial models of soot formation in flames (Frenklach 2002; Frenklach \& 
Wang 1991), was initially discounted as the main mechanism of carbon dust production (Cadwell et al. 1994; Cherchneff et al. 1992; Frenklach et al. 1989a). Astrophysical models were favoring high temperature nucleation of mineral particles in or near the photosphere, onto which PAH molecules could later condense (Cherchneff \& Cau 1998). In an attempt to assess alternative pathways of dust formation, which could be potentially competing, Cherchneff (2000) investigated the formation of van der Waals bound $\mathrm{PAH}$ dimers in the envelopes, considering species as small as benzene $\left(\mathrm{C}_{6} \mathrm{H}_{6}\right)$ and up to coronene $\left(\mathrm{C}_{24} \mathrm{H}_{12}\right)$. The conclusion was that PAH dimers could act as dust nuclei in the inner shells (Cherchneff 2000). Cau (2002) later on included periodic shocks in the model and inferred that the amount of PAHs and dimers produced, considering aromatics containing up to 7 rings, is not enough to explain the formation of carbon dust in the atmosphere of the IRC +10216 star, but that it can account for the formation of the small disordered cores of the kind observed in presolar grains (Cau 2002).

In this paper, we re-examine the condensation of PAH molecules in the envelopes of carbon rich stars based on high level theoretical calculations supported by state-of-the-art laboratory experiments related to the thermodynamics and kinetics of the process. Our findings are applied to the envelope of the evolved red giant, IRC +10216 .

\section{Combined experimental/theoretical approach}

Laboratory experiments have been conducted to identify the temperature range under which pyrene $\left(\mathrm{C}_{16} \mathrm{H}_{10}\right)$ molecules dimerize (Sabbah et al. 2010). Our experimental approach is briefly summarized below to provide the reader with some experimental background (Sabbah et al. 2010). The experiments were performed using a continuous flow CRESU apparatus (Canosa et al. 2008; Dupeyrat et al. 1985), adapted for condensable species such as PAHs (Goulay et al. 2006). This chemical reactor is designed to generate dense uniform flows using a Laval nozzle, over a wide range of temperatures $(60-470 \mathrm{~K})$ and containing high (supersaturated) concentrations of PAH vapors. The time evolution of the chemical species present in the reactor is monitored by a time of flight mass spectrometer (TOFMS) equipped with a VUV laser for photoionization of the neutral reagents and products. The onset of nucleation is explored for a set of flow temperatures. It is evidenced by the collapse of the PAH ion signal above a certain initial PAH concentration. At these high degrees of supersaturation, dimers may be considered as critical nuclei within the framework of classical nucleation theory. Once they are formed, they go on to react rapidly with more monomer to form trimers and larger clusters. After the identification of the temperature range over which nucleation is occurring, we proceed to kinetics measurements on dimerization within this range, but at lower PAH monomer concentrations. This yields the apparent second order rate coefficient for removal of $\mathrm{PAH}$ monomer, according to simple second-order kinetics.

The experimental results are coupled with theoretical calculations that employ careful consideration of the intermolecular interaction energies and intermolecular dynamics to estimate the binding energy, equilibrium constant, and rate coefficient 
(Sabbah et al. 2010). The theoretical analysis employs a sum of atom-atom potentials to describe the intermolecular interactions. The parameters in this potential are designed to reproduce the equilibrium binding energies from high level calculations for a range of small PAH dimers, while at the same time accurately modeling the interactions for the large separations that span the transition state region for the association. The analysis focuses on the rigid body dynamics of the two PAH molecules, and employs transition state theory and trajectory simulations to examine the high pressure addition kinetics. Predictions for the equilibrium constant are obtained from Monte Carlo integration of the classical phase integral representation of the intermolecular partition function. The intramolecular partition functions of the monomer are assumed to be invariant to the dimerization process. These quantitative coupled anharmonic predictions for the equilibrium constant are used in the following analysis.

\section{Astrophysical implications}

The stable cluster structures of PAHs ranging from pyrene $\left(\mathrm{C}_{16} \mathrm{H}_{10}\right)$ to circumcoronene $\left(\mathrm{C}_{54} \mathrm{H}_{18}\right)$ have been explored theoretically recently (Rapacioli et al. 2006). Stacking PAH molecules was found to yield the most stable motif. The atomistic model developed in that work was then used in molecular dynamics simulations to study the nucleation and evaporation of coronene $\left(\mathrm{C}_{24} \mathrm{H}_{12}\right)$ clusters. Rapacioli et al. (2006) found that under cold interstellar conditions, most of the collisions lead to cluster growth. They claimed that PAH clusters could represent the missing link between small carbonaceous grains and free isolated PAH molecules. Here we extend the exploration to the much warmer environment of the envelope of a late carbon star in which PAHs are supposedly generated and the existence of PAH clusters postulated. We first describe below the physical conditions characterizing the extensively studied, albeit not fully understood, envelope of IRC +10216 . This late carbon rich star is one of the brightest sources in the infrared sky as a result of thermal re-emission by dust.

The modeling of infrared astronomical observations, led Fonfria et al. to divide the envelope in concentric regions (Fonfria et al. 2008). They first distinguish the inner shell comprised between the stellar photosphere and about $5 R_{\star}$. The outer edge corresponds to the first grain formation zone. Radiation pressure on grains, together with momentum coupling of gas and grains, strongly accelerate the gas up to $v_{\exp }(r) \sim 11 \mathrm{~km} \mathrm{~s}^{-1}$. In the intermediate envelope, the gas expands adiabatically until it reaches $\sim 20 R_{\star}$ where it is strongly accelerated for a second time up to its terminal velocity $\sim 14.5 \mathrm{~km} \mathrm{~s}^{-1}$. The second acceleration zone corresponds to the second dust formation layer.

In this work, we adopt the radial kinetic temperature profile derived from the best fit of observed $\mathrm{C}_{2} \mathrm{H}_{2}$ lines (Fonfria et al. 2008) with an effective stellar temperature, $T_{\text {eff }} \sim 2330 \mathrm{~K}$. The temperature of molecules such as PAHs in the extended atmosphere is governed by balancing heating and cooling processes. PAH molecules are heated by (1) collisions with abundant $\mathrm{H}_{2}$ gas; and (2) absorption of central star radiation, which peaks in the near infrared. As small PAH molecules do 


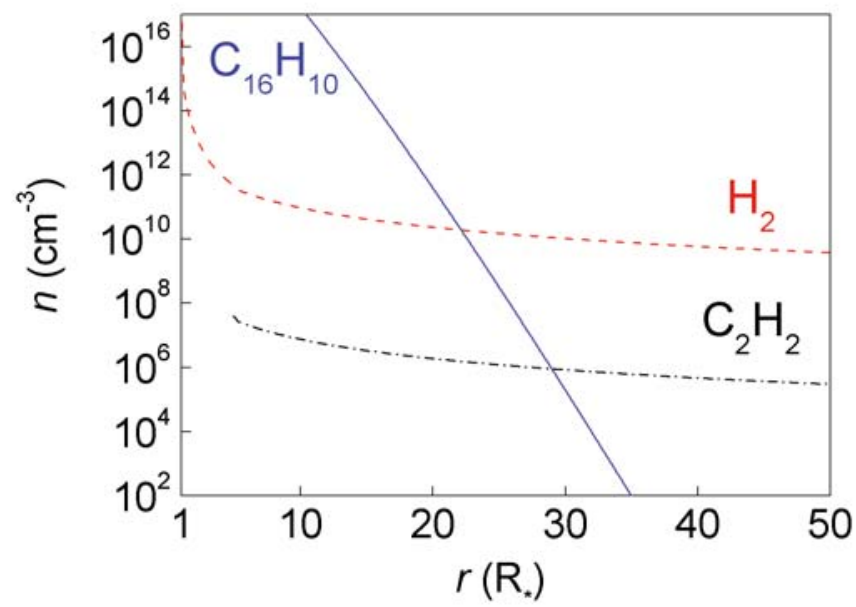

Fig. 1. Minimum pyrene abundance for condensing $10 \%$ of the monomers versus the distance from the central star IRC +10216 under a high molecular hydrogen density scenario from Agundez \& Cernicharo (2006). Molecular abundances of $\mathrm{H}_{2}$ (Agundez \& Cernicharo 2006) and $\mathrm{C}_{2} \mathrm{H}_{2}$ (Fonfria et al. 2008) are indicated for comparison. The condensation is governed by the PAH temperature which slowly decouples from the gas because of the high number of collisions. The difference between the gas kinetic temperature and the internal pyrene temperature initially null at the photosphere rises up to a maximum of $250 \mathrm{~K}$ at around $5 R_{\star}$ and then slowly decreases down to $200 \mathrm{~K}$ at $25 R_{\star}$.

not absorb efficiently in this energy range, heating is therefore mainly ensured by collisions with molecular hydrogen. Farther from the central star, the total density drops, collisions become less frequent and the vibrational temperature of the PAHs may then decouple from the kinetic temperature of the gas. The main cooling mechanism has been identified as cascade emission of infrared photons. We follow the approach developed by Cherchneff et al. to derive the vibrational temperature of pyrene. Adopting a spherical geometry and neglecting bipolar outflow cavities, the density profile, $n(r)$, is given by the law of conservation of mass and therefore depends linearly on the mass loss rate and the expansion velocity:

$$
\dot{M}=n(r) \mu m_{H} v_{\exp }(r) 4 \pi r^{2}
$$

with $m_{H}$, the mass of the hydrogen atom, and $\mu \sim 2$ assuming that all hydrogen is molecular. Following the work of Agundez (Agundez et al. 2006), we consider two different density profiles of the gas across the envelope hence bracketing the range of mass loss rates.

The equilibrium constant for pyrene dimerization (Sabbah et al. 2010) determined from the combined experimental/theoretical work is used to calculate the 


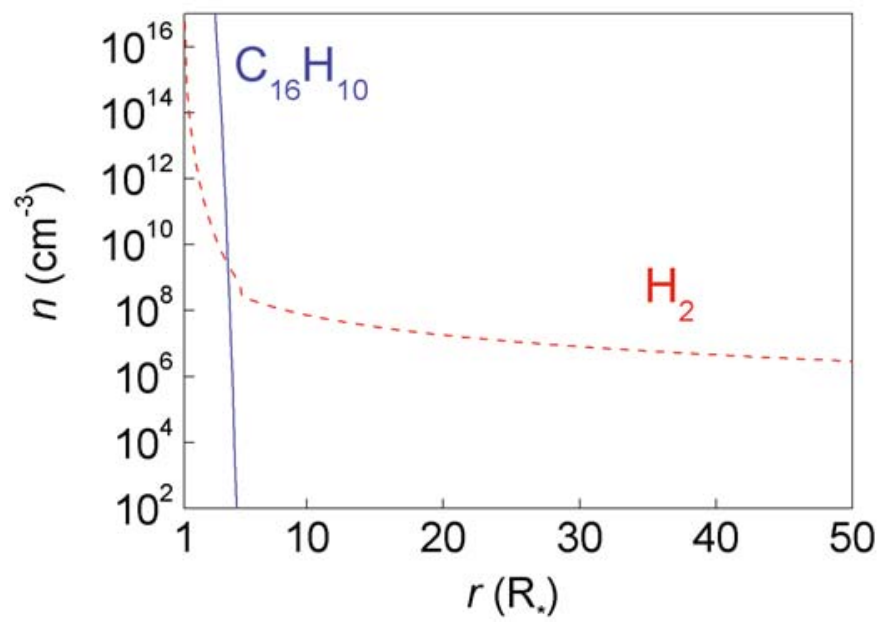

Fig. 2. Minimum pyrene abundance for condensing $10 \%$ of the monomers versus the distance from the central star IRC +10216 under a low molecular hydrogen density scenario from Agundez \& Cernicharo (2006). Molecular abundance of $\mathrm{H}_{2}$ (Agundez \& Cernicharo 2006) is indicated. The condensation is governed by the PAH temperature which decouples from the gas kinetic temperature very close to the star. At the distance of $5 R_{\star}$, the pyrene internal temperature has already dropped below $100 \mathrm{~K}$.

minimum PAH number density for condensing $10 \%$ of the PAH monomers at a given temperature. The resulting minimum abundances are plotted in Figures 1 and 2 along with the molecular hydrogen and acetylene density profiles.

The most striking feature is the high sensitivity of the minimum pyrene abundance to the total gas density. As soon as the gas density drops, collisions are not enough to keep the PAH molecules hot. The PAH vibrational temperature starts to decouple from the gas kinetics temperature, and therefore the association between two cooler PAH molecules becomes faster than the destruction of the dimer. When considering a high relative abundance of $10^{-9}$ for each of the PAHs, the plots show that condensation may occur $>5 R_{\star}$ and $35 R_{\star}$ under the low and high density scenarios, respectively. Based on these observations, we first conclude that under the high gas density scenario, condensation of small PAHs occurs too far from the star $\left(>35 R_{\star}\right)$ to represent a key mechanism in the formation of carbon dust particles.

The low gas density scenario seems more favorable. We look now at the kinetics of the association process to extract some time constraints. Collisions between cold PAH molecules above the edge of the inner shell lead to an energized complex which either relaxes by emission of infrared photons, relaxes by collision with a 
third body, or breaks apart. Calculations have revealed a surprising decoupling between the inter- and intra-molecular modes in the PAH complex (Sabbah et al. 2010). This means that under low pressure environments the excess collisional energy may not be that easily evacuated through infrared emission and may rather cause the complex to break apart, hence reducing its lifetime. Nevertheless, the hypothesis of a $100 \%$ sticking efficiency provides an upper limit to the lifetime of the PAH complex. A simple calculation then gives a lower limit for the growth time - corresponding to the inverse of the product of the capture rate and the $\mathrm{PAH}$ abundance - of $\sim 10^{9} \mathrm{~s}$. This characteristic growth time is 20 times longer than the period of the shock, $\sim 630$ days, that strongly affects the inner shell of the carbon star. Based on this kinetics argument, one can then conclude that the formation of PAH clusters is slow and will then be perturbed by the rise in temperature of the environment caused by the propagation of the shock.

\section{Conclusion}

We have re-examined the condensation of small PAHs in the envelope of IRC +10216 . As the distance from the star grows, the gas density drops and the temperature of the PAH molecules decouples from the gas kinetic temperature. Cold PAHs molecules are then more likely to stick to each other and form a dimer. The thermodynamics of pyrene dimers in this environment is explored using the equilibrium constant of the dimer derived from the combined experimental/theoretical work.

Our results show that a low density of gas is necessary for small PAHs such as pyrene to condense at the edge of the inner shell of the star. However, even then the growth of these clusters is expected to be slow and therefore seriously hindered by periodic shocks induced by stellar pulsations. This rules out clustering of small PAHs as the main mechanism for generation of carbon particles in these environments. Our conclusions do not contradict the findings of Bernatowitcz (2006) as heterogeneous condensation of carbonaceous molecules on carbides is still possible. Alternatively, larger PAHs could stack efficiently under conditions prevailing in high temperatures environments. The chemical pathway leading to these large aromatic units remains to be examined.

In the laboratory, there are a number of challenges to take up to explore the kinetics of dimerization of larger PAHs than pyrene. The most difficult one is probably to evaporate these condensable species and mix them with the buffer gas to produce a uniform continuous flow with a sufficient (and known) amount of reactant, stable over time. An alternative such as laser desorption is not suitable because, although the technique can gently vaporize molecules, the amount of molecules desorbed is unknown and it generally relies on pulsed laser systems.

Future studies will then rather extend the theoretical approach developed here for pyrene to larger species more prone to condensation.

A more complete study would include periodic shocks which boost the density (and therefore the number of collisions) and cause a rise in temperature of the 
medium. Other routes based on chemical growth should be explored by chemical kinetics models.

The authors acknowledge funding from the Indo-French Centre for the Promotion of Advanced Research (Project number 3405-3). This work is also supported by the French Programmes of Physique Stellaire and of Physique et Chimie du Milieu Interstellaire. The theoretical work (S.J.K.) has been supported by NASA's Planetary Atmospheres Program through grant NNH09AK24I and by the US Department of Energy, Office of Basic Energy Sciences, Division of Chemical Sciences, Geosciences, and Biosciences under Contract No. DE-AC02-06CH11357.

\section{References}

Agundez, M., \& Cernicharo, J., 2006, ApJ, 650, 374

Allain, T., Sedlmayr, E., \& Leach, S., 1997, A\&A, 323, 163

Bernatowicz, T., Croat, T.K., \& Daulton, T.L., 2006, MESS, 943, 109

Boersma, C., Hony, S., \& Tielens, A., 2006, A\&A, 447, 213

Cadwell, B.J., Wang, H., Feigelson, E.D., \& Frenklach, M., 1994, ApJ, 429, 285

Canosa, A., Goulay, F., Sims, I.R., \& Rowe, B.R., 2008, In Low Temperatures and Cold Molecules, ed. IWM Smith, 55 (Singapore: World Scientific)

Cau, P., 2002, A\&A, 392, 203

Cherchneff, I., Barker, J.R., \& Tielens, A., 1992, ApJ, 401, 269

Cherchneff, I., \& Cau, P., 1998, Proceedings of the Asymptotic Giant Branch Stars 191th IAU Symp., 251

Cherchneff, I., 2000, The Carbon Star Phenomenon, Proceedings of the 177th IAU Symp. Cherchneff, I., 2011, Proceedings of PAHs and the Universe Conference, this volume Dupeyrat, G., Marquette, J.B., \& Rowe, B.R., 1985, In Physics of Fluids, 1273

Fonfria, J.P., Cernicharo, J., Richter, M.J., \& Lacy, J.H., 2008, ApJ, 673, 445

Frenklach, M., 2002, Physical Chemistry Chemical Physics, 4, 2028

Frenklach, M., Carmer, C.S., \& Feigelson, E.D., 1989, Nature, 339, 196

Frenklach, M., \& Feigelson, E.D., 1989, ApJ, 341, 372

Frenklach, M., \& Wang, H., 1991, Proceedings of the Combustion Institute, 23, 1559

Goulay, F., Rebrion-Rowe, C., Biennier, L., et al., 2006, JPC A, 110, 3132

Guelin, M., Lucas, R., \& Cernicharo, J., 1993, A\&A, 280, L19

Henning, T., \& Salama, F., 1998, Science, 282, 2204

Jager, C., Huisken, F., Mutschke, H., Jansa, I.L., \& Henning, T.H., 2009, ApJ, 696, 706

Jager, C., Mutschke, H., Henning, T., \& Huisken, F., 2011, Proceedings of PAHs and the Universe Conference, this volume

Jones, A.P., 2005, In Proceedings of the Dusty and Molecular Universe: a Prelude to Herschel and Alma, ed. A Wilson, 239 (Ag Noordwijk: Esa Publications Division C/O Estec), 2200

McCabe, E.M., 1982, MNRAS, 200, 71

Rapacioli, M., Calvo, F., Spiegelman, F., Joblin, C., \& Wales, D.J., 2005, JPC A, 109, 2487

Sabbah, H., Biennier, L., Klippenstein, S.J., Sims, I.R., \& Rowe, B.R., 2010, JPC Lett., 2962

Tielens, A., 2008, ARA\&A, 46, 289

Ziurys, L.M., 2006, PNAS, 103, 12274 